Cite this: RSC Adv., 2013, 3, 22900

\title{
A foam-templated approach for fabricating organogels using a water-soluble polymer $\dagger$
}

\author{
Ashok R. Patel, ${ }^{\text {*a }}$ Domien Schatteman, ${ }^{a}$ Ans Lesaffer ${ }^{b}$ and Koen Dewettinck ${ }^{a}$
}

Received 29th August 2013

Accepted 25th September 2013

DOI: $10.1039 / \mathrm{c} 3 \mathrm{ra} 44763 \mathrm{~d}$

www.rsc.org/advances

We report a unique approach of using a water soluble polymer (a cellulose derivative) to generate organogels via a facile, low temperature process. This finding is an important step towards the development of liquid oil-based soft matter systems for applications in non-bio and bio-related fields.

In the last decade or two, organogelation or gelling of oils and organic solvents has received considerable attention from researchers representing diverse disciplines ranging from colloid and material science (nanostructure synthesis, energy transfer and light harvesting, optoelectronics) ${ }^{1}$ to bio-related fields such as cosmetics, foods, drug delivery, and tissue engineering. ${ }^{2}$ Much effort has been devoted to advance this field of research by synthesizing or designing new gelator molecules. ${ }^{3}$ However, even though a lot of reports have been published on both low molecular weight and polymer gelators, ${ }^{4}$ it is still hard to establish a rational strategy of designing such molecules. Moreover, these molecules require significant synthetic efforts and most importantly, they are of limited use in bio-related fields which severely undermines their universal applications.

Another area of study which has also received equal attention includes the study of organogelation property of known materials which are hydrophobic in nature such as waxes, phytosterols, 12-hydroxy stearic acid and hydrophobic polymers such as cellulose ethers. ${ }^{5}$ Each of these materials have some drawbacks including, but not limiting to waxy greasy taste, requirement of high concentration, not safe for use in humans and requirement of a high temperature process and additives to form organogels. Thus, if a facile method to prepare organogels without the use of any organic synthetic procedures and with the use of known \& safe materials using a simple low

${ }^{a}$ Vandemoortele Centre, Faculty of Bioscience Engg., Ghent University, Belgium. E-mail: Patel.Ashok@Ugent.be; Fax: +32 (0)9264 6218; Tel: +32 (0)9264 6209

${ }^{b}$ Vandemoortele $R \&$ D Centre, Prins Albertlaan 79, 8870 Izegem, Belgium

$\dagger$ Electronic supplementary information (ESI) available. See DOI: $10.1039 / \mathrm{c} 3 \mathrm{ra} 44763 \mathrm{~d}$ temperature process was developed, then one can broaden the practical applications of organogelators significantly.

In this paper, we report for the first time a new approach to gel liquid oil using a porous cryogel obtained from a watersoluble polymer through foam-templating. Hydroxy propyl methylcellulose (HPMC), a water soluble cellulose derivative, is a well-known industrial polymer which is also approved for pharmaceutical and foods applications. We exploited the surface activity of HPMC to form and stabilize an aqueous foam which was subjected to freeze drying to remove water, resulting in a formation of porous cryogel. Surprisingly, this porous material showed excellent oil sorption property, absorbing close to 100 times its weight of oil. This oil-sorbed porous material when sheared, gave us an excellent organogel with interesting rheological properties. Fig. 1 gives a step by step representation of organogel formation using this new approach.

Cellulose derivatives such as HPMC and methylcellulose are synthesized by substituting hydroxyl group of cellulose with hydroxyl propyl and methyl group. The substitution adds hydrophobic character to the molecules and thus they show an amphiphilic behaviour and certain degree of surface activity. ${ }^{6}$ Due to this surface activity, it was easy to incorporate air into
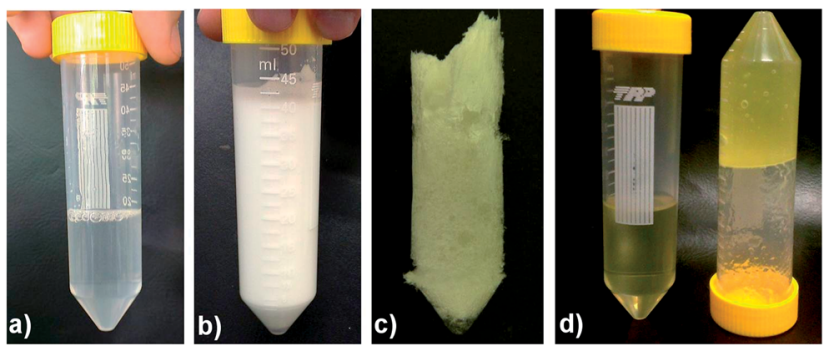

Fig. 1 Photographs of (a) 1 wt\% HPMC (4000 cps) solution; (b) aqueous foam formed by aerating HPMC solution; (c) porous cryogel obtained by removal of water by freeze drying and (d) comparative pictures of liquid oil (sunflower oil) on the left and organogel (with 98 wt\% liquid oil) formed using cryogel on the right (also refer to Fig. $\mathrm{S} 1 \dagger$ ). 
HPMC solution resulting in an overrun of over $300 \%$ depending on the viscosity grade of HPMC used. As expected, the low viscosity grade HPMC gave high overrun as compared to the higher viscosity grades. On the other hand, the susceptibility of foam to drainage and disproportionation was lower for the higher viscosity grades (refer Table S1 for experimental values $\dagger$ ). The foam stabilization property of cellulose derivatives is attributed to the gelled film formation of polymer at the airwater interface ${ }^{6}$ and hence, a higher viscosity grade polymer is expected to stabilize foam more efficiently as compared to a lower viscosity grade due to the formation of a interfacial film of a higher stiffness. Since, stabilization of foam is a prerequisite to porous cryogel formation, we selected highest viscosity grade (4000 cps) HPMC for the rest of the study.

Microstructures of aqueous foam, cryogel and organogel studied using optical, confocal and electron microscopy are presented in Fig. 2. Since, the aeration was carried out using a high-performance dispersing instrument (Ultraturrax ${ }^{\circledR}$ ) operating at $11000 \mathrm{rpm}$, the aqueous foam generated was very fine with an average bubble size (obtained from optical microscopy images) of less than $150 \mu \mathrm{m}$. The removal of water through freeze drying, resulted in a porous cryogel as seen in Fig. $2 \mathrm{~b}$. The voids or foam cells in the porous materials were non-uniform and the dimension was generally more than $200 \mu \mathrm{m}$ which is larger than the size of air bubbles in the aqueous foam, this is a characteristic microstructure of cellulose-based solid foams prepared through physical foaming. ${ }^{7}$ The difference in the dimension can be explained by the gas expansion induced foam destabilization (Ostwald ripening and coalescence) ${ }^{8}$ that might occur during the long drying time (48 hours) in the lyophilizer.
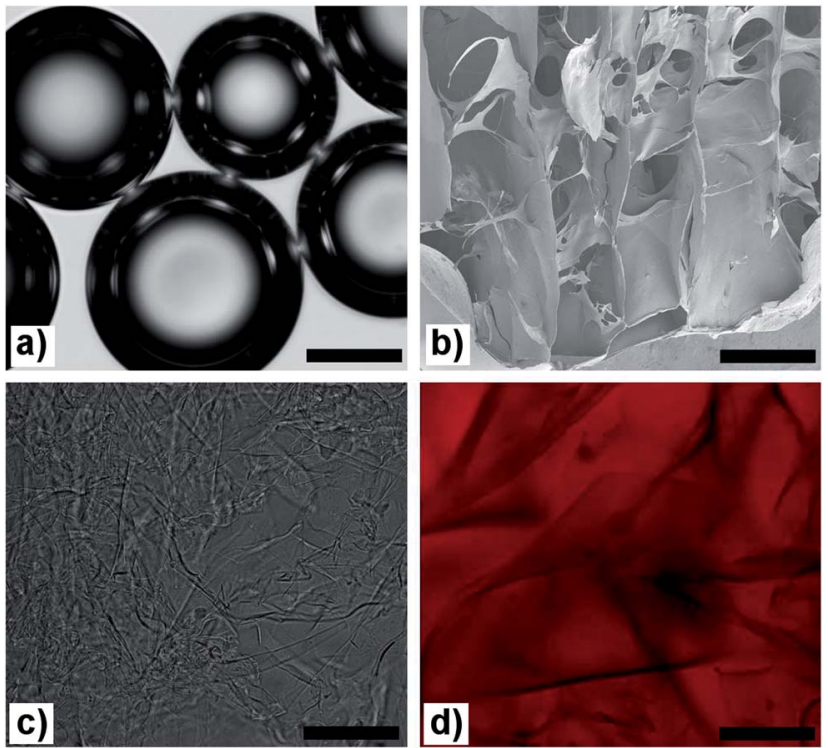

Fig. 2 (a) Optical microscopy image (scale bar $=50 \mu \mathrm{m}$ ) of aqueous foam generated from 1 wt\% solution of HPMC 4000 cps; (b) cryo-scanning electron microscopy image (scale bar $=100 \mu \mathrm{m}$ ) of porous cryogel obtained by freeze drying the aqueous foam; (c \& d) transmission and confocal microscopy images (scale bars $=100$ and $25 \mu \mathrm{m}$ respectively) of sunflower oil organogel formed at 2 wt\% polymer. Notice the un-stained polymer sheets dispersed in dyed oil continuous phase.
This porous material surprisingly showed excellent oil absorption property with a high swelling capacity (absorbing oil at 98 times its own weight within few seconds). A series of pictures taken every 60 seconds (Fig. 3) clearly indicates that the porous material showed a quick absorption of oil. Though watersoluble, HPMC and methylcellulose are known to have some affinity for oil as indicated by their use in stabilizing oil in water emulsions where they are known to adsorb at the oil-water interfaces. ${ }^{9}$ The oil affinity of these water soluble cellulose derivatives probably comes from the hydrophobic substituents (hydroxyl propyl and methyl groups) attached to cellulose molecule. However, since they cannot be simply dispersed in oil at room temperature, researchers have used high temperatures (usually above the glass transition temperature, $T_{\mathrm{g}}$ ) to get their oil dispersions usually in combination with a hydrophobic cellulose derivative such as ethylcellulose. ${ }^{5 \boldsymbol{d}, 10}$ In yet another attempt, the dispersibility of water-soluble cellulose derivatives was made possible by chemical modification i.e. functionalization of their - $\mathrm{OH}$ groups with 1,6-hexamethylene diisocyanate. ${ }^{\mathbf{1 1}}$ However, these attempts have severe practical limitations because of the use of very high temperatures (as high as $180{ }^{\circ} \mathrm{C}$ ) and requirement of chemical synthesis. Thus, the low-temperature process used in our current work, offers a pragmatic solution in overcoming these drawbacks.

As the cryogel was formed merely by using HPMC polymer without any crosslinking, it showed absorbent property of a reticulated solid foam (with open cells) ${ }^{7 \boldsymbol{b}}$ i.e. the oil could easily flow through the entire structure and hence be quickly absorbed but not tightly bound and thus could be oozed out under minimum pressure. In order to prevent release of oil under pressure, we sheared this oil-sorbed material to uniformly disperse the polymer sheets in the oil continuous phase, resulting in the formation of oil gel where the oil was physically trapped in the network of dispersed polymer sheets. The microstructure of organogel prepared using $98 \mathrm{wt} \%$ oil and $2 \mathrm{wt}$ $\%$ polymer as seen in Fig. $2 \mathrm{c}$ and d, confirms the sheet of polymer dispersed uniformly throughout the oil continuous phase (please also refer to Fig. $\mathrm{S} 2 \uparrow$ for the volume view generated from stacks of confocal images). The polymer sheets were also imaged under polarized light (Fig. S3†) and as expected they were mostly non-crystalline in nature.

Organogels with viscosity $(\eta)$ of up to $6000 \mathrm{~Pa} s$ could be obtained at a concentration of $5 \mathrm{wt} \%$ polymer; there was a decrease in the viscosity with the decrease in the polymer concentration with values falling below $400 \mathrm{~Pa} s$ at $1 \mathrm{wt} \%$ polymer (Fig. 4a). Due to this increase in the viscosity, it was observed that at concentrations above $2 \mathrm{wt} \%$ polymer, the oil-

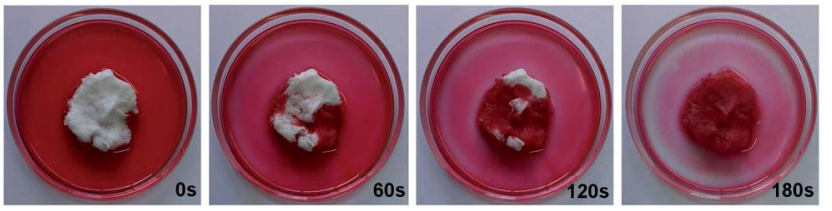

Fig. 3 A series of photographs displaying the quick oil absorption by porous cryogel. Sunflower oil with Nile red dye was used for clear representation and images were captured every 60 seconds. 
sorbed material was comparatively difficult to shear and took longer time for homogenization. Hence, we selected 2 wt $\%$ polymer as the concentration for rest of our study. To study the behaviour of organogel under dynamic shear, a series of oscillatory measurements (including amplitude, temperature and frequency sweeps) were carried out on organogel sample prepared using 2 wt $\%$ polymer. The complex modulus, $G^{*}$ (the measure of the total resistance of the sample to applied deformation) plotted against oscillatory stress (Fig. S4†) shows that the sample has a narrow linear viscoelastic region (LVR). The progressive increase of phase angle $(\delta)$ and a final value reaching close to 80 degrees suggests that there is a gel-sol transformation when the dynamic stress is increased on the sample. Further, we observed a prominent cross-over point where $G^{\prime \prime}$ (loss modulus) crosses over $G^{\prime}$ (storage modulus) at an oscillatory stress of $129.7 \mathrm{~Pa}$ (Fig. 4b) indicating a gel-sol transformation. This results suggests that the gel is kept together by rather weak internal forces which are overcome by higher applied stress. However, the gel stiffness, $G^{\prime}$ at LVR region was in the range of $10^{4}$, which is similar to the edible oil organogels prepared using a combination of $5 \mathrm{wt} \%$ ethylcellulose and $5 \mathrm{wt}$ $\%$ surfactant (sodium stearoyl lactylate) via a high temperature process. ${ }^{12}$ Thus, these results suggests that this low temperature process can be effectively utilized to generate organogels with similar rheological properties. The evolution of $G^{\prime}$ and $G^{\prime \prime}$ with frequency (Fig. 4c) is very similar to a solid-like gel material, $G^{\prime}$ values were always higher than $G^{\prime \prime}$ values in the whole frequency range studied, and the "plateau" region of the mechanical spectrum was noticed, i.e. $G^{\prime}$ increases slightly with frequency
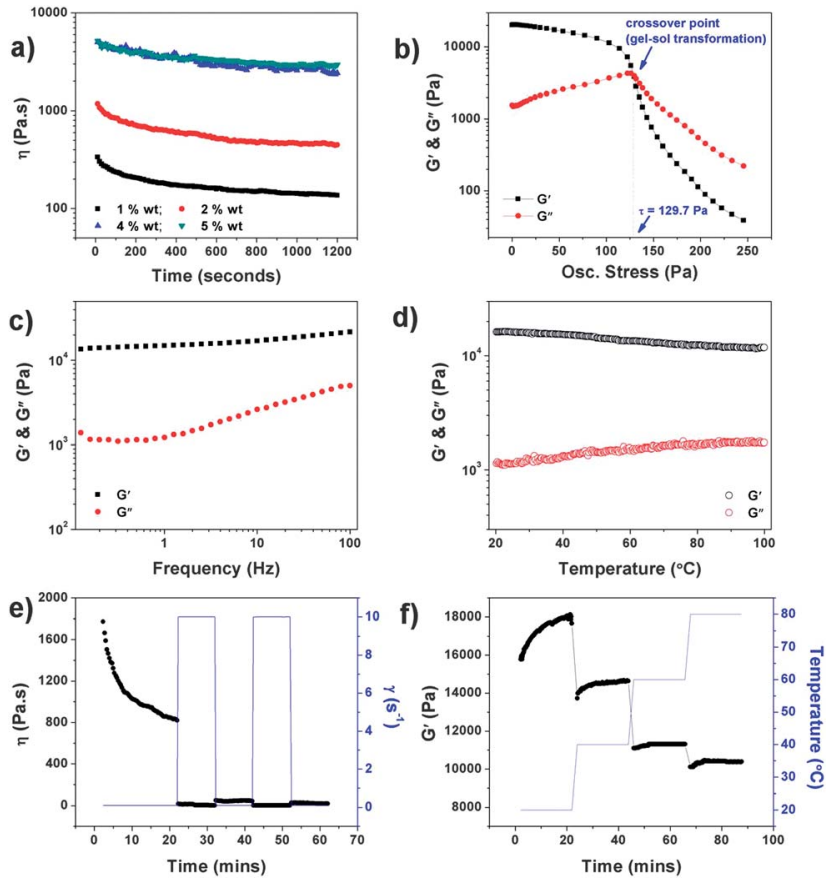

Fig. 4 (a) Viscosity curves of organogels prepared at varying concentration of polymer; (b-d) amplitude, frequency and temperature sweeps carried out on organogel sample prepared at 2 wt\% polymer; (e) viscosity changes followed in time against alternating low $\left(0.1 \mathrm{~s}^{-1}\right)$ and high $\left(10 \mathrm{~s}^{-1}\right)$ shear rates; $(f) G^{\prime}$ versus time at varying temperatures. and $G^{\prime \prime}$ displays a minimum at intermediate frequencies. Further, the temperature response of organogels as shown in Fig. $4 \mathrm{~d}$ indicates that the $G^{\prime}$ of organogels remains around $10^{4}$ even at higher temperatures $\left(100^{\circ} \mathrm{C}\right)$ with around one order of magnitude higher than $G^{\prime \prime}$. This temperature related mechanical spectrum corresponds with the traditional description given for lithium-based lubricating greases. $^{\mathbf{5 d , 1 0}}$ Thus, this finding could also be of particular interest to researchers working in the field of bio-lubricating greases for identification of non-toxic leads.

The organogels showed thixotropic behaviour resulting in the loss of viscosity over time at constant shear rate. As seen in Fig. 4e, viscosity values drop from an initial $\sim 1800 \mathrm{~Pa}$ s to $\sim 800$ $\mathrm{Pa} \mathrm{s}$ within 20 minutes even when sheared at a lower rate $\left(0.1 \mathrm{~s}^{-1}\right)$. The system also showed a strong shear-thinning behaviour with more than a 40 -fold drop in viscosity from around 820 to $17 \mathrm{~Pa} \mathrm{~s}$ on increasing the shear rate from 0.1 to $10 \mathrm{~s}^{-1}$. As further seen from the figure, there was a negligible structure recovery when the shear rate was changed back to $0.1 \mathrm{~s}^{-1}$. Currently we are evaluating the possibility of using small molecular weight molecules such as monoglycerides to work together with HPMC and influence the structure recovery.

The temperature-time related behaviour of organogel however was very interesting. The gel stiffness, $G^{\prime}$ was measured over time at increasing temperatures and as seen from Fig. $4 \mathrm{f}$, the $G^{\prime}$ had a value of above $10000 \mathrm{~Pa}$ even at temperature as high as $80{ }^{\circ} \mathrm{C}$, suggesting that the solid-gel like behaviour of organogel was maintained even at higher temperatures under dynamic shear. This is a clear advantage over the organogels prepared using waxes as gelators where we see a complete loss of structure above $40{ }^{\circ} \mathrm{C}$ because of the melting of crystals. ${ }^{5 a}$

Moreover, the organogel samples stored at $5{ }^{\circ} \mathrm{C}$ for over 3 months showed no signs of oiling-out or polymer sedimentation (Fig. S5†) indicating good storage stability of these systems.

\section{Conclusions}

In conclusion, we successfully demonstrated a facile approach of making organogels of liquid oil using a water-soluble polymer. The novelty of this method lies in the simplicity of preparation which only involves eco-friendly steps like aqueous foaming, freeze drying, and shearing without the use of any high temperatures, additional additives, harsh chemicals or crosslinkers. The rheological characterization confirmed that the organogels prepared by this approach had interesting properties which are comparable or in some cases better than organogels (such as ethylcellulose, wax, conventional grease etc.) previously reported in the literature. Hence, this finding should generate interest among researchers working in both non-bio as well as bio-related fields and the finding also warrants further research in establishing this approach for exploring other water-soluble polymers with good foaming properties (such as gelatin, hydrophobically modified starch etc.) to act as organogelators.

This research is supported by Marie Curie Career Integration Grant within the 7th European Community Framework Programme. 


\section{Notes and references}

1 (a) D. J. Abdallah, L. Lu and R. G. Weiss, Chem. Mater., 1999, 11, 2907-2911; (b) D. J. Abdallah and R. G. Weiss, Adv. Mater., 2000, 12, 1237-1247.

2 (a) Edible oleogels: Structure and health implications, AOCS Press, Urbana, Illinois, 2011; (b) G. Bastiat and J.-C. Leroux, J. Mater. Chem., 2009, 19, 3867-3877; (c) F. Aparicio, E. Matesanz and L. Sanchez, Chem. Commun., 2012, 48, 5757-5759; (d) H. Svobodova, V. Noponen, E. Kolehmainen and E. Sievanen, RSC Adv., 2012, 2, 4985-5007; (e) A. I. Romoscanu and R. Mezzenga, Langmuir, 2006, 22, 7812-7818.

3 (a) C. Kang, Z. Bian, Y. He, F. Han, X. Qiu and L. Gao, Chem. Commun., 2011, 47, 10746-10748; (b) M. Suzuki, H. Saito, H. Shirai and K. Hanabusa, New J. Chem., 2007, 31, 16541660.

4 (a) M. Suzuki and K. Hanabusa, Chem. Soc. Rev., 2009, 38, 967-975; (b) M. Suzuki and K. Hanabusa, Chem. Soc. Rev., 2010, 39, 455-463.

5 (a) A. R. Patel, D. Schatteman, W. H. D. Vos and K. Dewettinck, RSC Adv., 2013, 3, 5324-5327; (b) M. Pernetti, K. F. van Malssen, E. Flöter and A. Bot, Curr. Opin. Colloid Interface Sci., 2007, 12, 221-231; (c) A. R. Patel, C. Remijn, P. C. M. Heussen, R. den Adel and K. P. Velikov, ChemPhysChem, 2013, 14, 305-310; (d) R. Sanchez, J. M. Franco, M. A. Delgado, C. Valencia and C. Gallegos, Green Chem., 2009, 11, 686-693; (e) A. R. Patel,
D. Schatteman, W. H. D. Vos, A. Lessafer and K. Dewettinck, J. Colloid Interface Sci., 2013, DOI: 10.1016/ j.jcis.2013.08.039.

6 (a) T. Shibata, in Renewable Resources for Functional Polymers and Biomaterials: Polysaccharides, Proteins and Polyesters, The Royal Society of Chemistry, 2011, pp. 48-87; (b) A. R. Patel, E. Drost, T. B. J. Blijdenstein and K. P. Velikov, ChemPhysChem, 2012, 13, 3777-3781; (c) A. R. Patel, J. Seijen ten-Hoorn, J. Hazekamp, T. B. J. Blijdenstein and K. P. Velikov, Soft Matter, 2013, 9, 1428-1436.

7 (a) S.-T. Lee and N. S. Ramesh, Polymeric Foams: Mechanisms and Materials, CRC Press, Boca Raton, Fla, 2004; (b) N. Brun, S. Ungureanu, H. Deleuze and R. Backov, Chem. Soc. Rev., 2011, 40, 771-788.

8 A. Colin, in Foam Engineering, John Wiley \& Sons, Ltd, 2012, pp. 75-90.

9 K. Kamide, Cellulose and Cellulose Derivatives: Molecular Characterization and it Applications, Elsevier B.V., Amsterdam, The Netherlands, 2005.

10 R. Sánchez, J. M. Franco, M. A. Delgado, C. Valencia and C. Gallegos, J. Ind. Eng. Chem., 2011, 17, 705-711.

11 (a) R. Gallego, J. F. Arteaga, C. Valencia and J. M. Franco, Cellulose, 2013, 20, 495-507; (b) R. Gallego, J. F. Arteaga, C. Valencia and J. M. Franco, Carbohydr. Polym., 2013, 98, 152-160.

12 T. Dey, D. A. Kim and A. G. Marangoni, in Edible Oleogels: Structure and Health Implications, ed. A. G. Marangoni and N. Garti, AOCS Press, Urbana, Illinois, 2011, pp. 295-312. 